\title{
Comportamento de monta e características seminais de suínos jovens landrace e large white
}

\author{
Mating behavior and seminal characteristics in young landrace and large white boars
}

\author{
Fabiane Mendonça Ferreira ${ }^{1}$ Ivo Wentz ${ }^{2}$ Isabel Regina Scheid ${ }^{3}$ Simone Bonini Afonso $^{4}$ \\ Antônio Lourenço Guidoni ${ }^{5}$ Fernando Pandolfo Bortolozzo ${ }^{2}$
}

RESUMO

O objetivo deste trabalho foi avaliar o comportamento de monta e as características seminais de machos Landrace (LD) e Large White (LW) dos 143 aos 232 dias de idade. A partir dos 143 dias de idade, 62 machos suínos foram avaliados quanto ao comportamento de monta. A partir dos 150 dias de idade, o sêmen foi coletado de 33 desses machos durante onze semanas para avaliar a produção e qualidade espermáticas. Não houve diferenças entre as raças em relação ao comportamento de monta $(p=0,105)$. Durante o período de coletas de sêmen, houve aumento do volume de sêmen, da concentração espermática, do número de espermatozóides (SPTZ) ejaculados e da liberação diária de SPTZ (LDE). O percentual de células espermáticas anormais diminuiu durante o período e não houve diferença entre as raças $(P=0,523)$. Os machos $L D$ ejacularam maior volume de sêmen $(P=0,016)$, de espermatozóides totais por ejaculado $(P=0,070)$ e apresentaram maior LDE $(P=0,042)$. Esses dados indicam que machos LD apresentam maior produção espermática e maior potencial para produzir doses de sêmen no período pós-púbere.

Palavras-chave: macho suíno, reprodução, produção espermática, idade, raça.

\section{ABSTRACT}

The aim of this work was to evaluate mating behavior and seminal characteristics of Landrace (LD) and Large White (LW) boars from 143 to 232 days of age. With 143 days of age, $L D(n=28)$ and $L W(n=34)$ boars were evaluated in relation to mating behavior. Being 150 days of age, semen was collected from 33 of these males during eleven weeks to evaluate sperm production and quality. No difference was observed between breeds in relation to mating behavior. During the period of semen collection, there was an increase in semen volume, sperm concentration and motility, total sperm in ejaculate, and in daily sperm output (DSO). The percent of abnormal sperm cells decreased during the period and there was no difference between breeds $(P=0.523)$. The $L D$ boars ejaculated higher semen volume $(P=0,016)$, total sperm in ejaculate $(P=0.070)$, and showed higher DSO $(P=0.042)$. These data indicate that LD boars show higher sperm production, and higher potential to produce semen doses in post pubertal period.

Key words: boar, reproduction, sperm production, age, breed.

\section{INTRODUÇÃO}

Uma parcela importante da eficiência econômica da atividade suinícola é atribuída ao desempenho reprodutivo dos machos. Devido aos avanços obtidos nos últimos anos em programas de melhoramento genético, machos destinados à reprodução apresentam taxas de ganho de peso superiores a $750 \mathrm{~g} \mathrm{dia}^{-1}$, sendo capazes de atingir os

\footnotetext{
${ }^{1}$ Médico Veterinário, Doutor, Pesquisador do Pólo de Inovação Tecnológica do RS, Universidade de Cruz Alta (UNICRUZ), Rua Andrade Neves, 308, 98000-000, Cruz Alta, RS. E-mail: fabianemf@hotmail.com. Autor para correspondência.

${ }^{2}$ Médico Veterinário, Doutor, Professor Adjunto, Faculdade de Veterinária, Setor de Suínos, Universidade Federal do Rio Grande do Sul (UFRGS).

${ }^{3}$ Médico Veterinário, Doutor, Pesquisadora do Centro Nacional de Pesquisa de Suínos e Aves (CNPSA), Empresa Brasileira de Pesquisa Agropecuária (EMBRAPA).

${ }^{4}$ Médico Veterinário,Mestre, Autônomo.

${ }^{5}$ Agrônomo, Doutor em Estatística, pesquisadora do CNPSA, EMBRAPA.
} 
$100 \mathrm{~kg}$ antes dos cinco meses de idade. O desenvolvimento corporal desses animais pode sugerir, em muitos casos, a possibilidade de antecipação da vida reprodutiva.

A norma que orienta o manejo de machos jovens preconiza a entrada em serviço em torno dos oito meses de idade (SCHEID \& AFONSO, 2000). Essa recomendação foi elaborada com base nos dados fisiológicos obtidos em trabalhos realizados principalmente entre as décadas de 60 e 80 em rebanhos europeus (SWIERSTRA, 1968a; SWIERSTRA \& DYCK, 1976; FLORCRUZ \& LAPWOOD, 1978; CAMERON, 1987) e com animais que apresentavam desempenhos de crescimento inferiores aos observados na suinocultura moderna. A habilidade de montar e produzir ejaculados férteis são componentes importantes na decisão de utilizar um macho na reprodução. No entanto, informações a respeito da fisiologia reprodutiva de machos suínos utilizados em programas de melhoramento genético de rebanhos nacionais são escassas. A hipótese deste trabalho é que machos LD e LW jovens apresentem diferenças no que se refere ao comportamento reprodutivo e características seminais. O objetivo do presente trabalho foi estudar o comportamento de monta e as características seminais de machos LD e LW no período pós-púbere.

\section{MATERIAL E MÉTODOS}

\section{Animais e tratamentos}

Sessenta e dois machos foram sorteados de um grupo de aproximadamente 800 machos pertencentes ao plantel de uma unidade de melhoramento genético de suínos de agroindústria localizada no sul do Brasil. Os animais foram distribuídos em dois tratamentos de acordo com a raça (T1: LD, n=28 e T2: $L W, n=34)$ e submetidos a um teste para avaliação do comportamento de monta. Após, 33 destes animais (T1: LD, n=17 e T2: $\mathrm{LW}, \mathrm{n}=16$ ) foram utilizados para a avaliação das características espermáticas, durante onze semanas consecutivas de coleta de sêmen.

\section{Alojamento e manejo}

Os machos foram desmamados, em média, com 28 dias de idade, sendo transferidos para baias coletivas até atingirem $100 \mathrm{~kg}$ de peso corporal. Durante o período experimental, permaneceram alojados individualmente em baias de $2,5 \mathrm{~m}^{2}$.

\section{Delineamento experimental}

Os animais foram distribuídos ao acaso em baias individuais e procederam-se avaliações do comportamento de monta e de parâmetros seminais e espermáticos durante onze semanas consecutivas.

\section{Estudo 1: avaliação do comportamento de monta}

Ao atingirem $100 \mathrm{~kg}$ de peso corporal e idade média de 143 dias, 28 machos LD e 34 LW foram testados para avaliação do comportamento de monta. Em baia de $3 \times 4 \mathrm{~m}$, cada macho foi colocado na presença de uma fêmea em estro por 15 minutos. A cada apresentação, registrava-se o número de saltos corretos e incorretos realizados sobre a fêmea. O teste era dado por concluído no momento em que o macho saltava corretamente sobre a fêmea e demonstrava viabilidade de executar a cópula. Não se permitia, no entanto, a complementação do ato. Foi estabelecido o número máximo de dez exposições à fêmea, com intervalos variáveis de 1 a 3 dias entre as apresentações.

Estudo 2: avaliação longitudinal das características espermáticas

Regime de coleta de sêmen

Dezessete machos LD e $16 \mathrm{LW}$, previamente submetidos ao teste para avaliação do comportamento de monta, foram sorteados e treinados em manequim fixo para a coleta de sêmen pelo método da mão enluvada. Foi estabelecido o regime de duas coletas semanais de sêmen com intervalos regulares de 3 e 4 dias entre as coletas durante o período de onze semanas consecutivas.

Os animais da raça LD apresentavam em

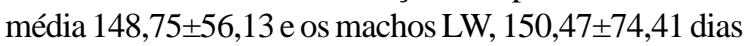
de idade na primeira coleta de sêmen.

\section{Avaliação do sêmen}

A cada coleta, foi determinado o volume de sêmen e motilidade espermática. Foram retiradas amostras de todos os ejaculados para a contagem do número de células espermáticas em câmara hemocitométrica (Neubauer) para estimar a concentração espermática. O número total de espermatozóides foi calculado através da multiplicação da concentração espermática/ml de sêmen pelo volume total ejaculado a cada coleta de sêmen. O exame da morfologia espermática foi realizado uma vez por semana, sob microscopia de contraste de fase em 1000 aumentos. As alterações da morfologia espermática foram classificadas em defeitos de peça intermediária, colo, defeitos de cauda, cauda em laço, gota protoplasmática proximal, gota protoplasmática distal, espermatozóides teratológicos e com defeitos de 
cabeça. Foram classificados como defeitos de cabeça tanto as alterações de tamanho e conformação, como as alterações de acrossoma. As alterações foram expressas em porcentagem.

\section{Análise estatística}

Para a comparação do número de montas corretas e incorretas utilizou-se o teste de quiquadrado. Para a análise das demais variáveis foi utilizada análise de variância através do procedimento GLM (SAS, 1998). Para o volume de sêmen, a motilidade, a concentração espermática, o número total de espermatozóides ejaculados e a morfologia espermática utilizou-se análise de variância para amostras repetidas considerando-se o efeito da raça e da semana de coleta. As médias foram comparadas pelo teste T de Student.

\section{RESULTADOS}

Estudo 1

Todos os animais saltaram sobre as fêmeas e somente dois não realizaram a monta correta. Os machos LD e LW apresentavam, em média, 145 dias de idade quando efetuaram a monta correta, não sendo observada diferença quanto ao comportamento de monta entre as raças $(p=0,105)$.

Estudo 2

O volume de sêmen ejaculado aumentou gradualmente durante o período experimental, não sendo observada uma tendência à estabilização (Figura 1). Analisando os valores médios durante as onze semanas de coleta, os animais da raça LD $(195,1 \mathrm{ml})$ apresentaram volumes de sêmen superiores $(\mathrm{P}=0,0157)$ aos LW (176,9ml).

Quanto à concentração espermática, foi observado o aumento gradual durante as primeiras sete semanas de coleta de sêmen. A partir da oitava semana, foram observados valores médios relativamente constantes, não havendo diferença significativa $(\mathrm{P}=0,668)$ entre as raças $\mathrm{LD}(164,76 \pm 13,88$ x $10^{6}$ espermatozóides $\left.\mathrm{ml}^{-1}\right)$ e LW $\left(155,99 \pm 14,31\right.$ x $10^{6}$ espermatozóides $\mathrm{ml}^{-1}$ ).

Na figura 2, é possível observar que os machos LD ejacularam espermatozóides em contagens

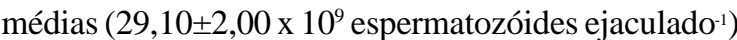
significativamente superiores aos LW $\left(23,72 \pm 2,06 \times 10^{9}\right.$ espermatozóides ejaculado $\left.{ }^{-1}\right)(\mathrm{P}=0,070)$. Foi observada a tendência de estabilização do número de espermatozóides ejaculados a partir da sétima semana de coleta, para os machos LD e da oitava semana para os LW. A partir da sétima semana, foi observado o estabelecimento de diferença significativa entre as duas raças, que permaneceu até a última coleta de sêmen. Foi possível observar que os animais da raça LD apresentaram valor médio superior aos da raça LW no que se refere à liberação diária de espermatozóides $(\mathrm{P}=0,0482$, figura 3$)$.

Em relação à motilidade das células espermáticas, foi observado o aumento gradual até a sétima semana, seguido de estabilização. Não houve diferença significativa na comparação entre as raças $\mathrm{LD}$ e $\mathrm{LW}(\mathrm{P}=0,315)$, cujos valores médios de onze

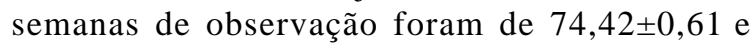
$75,43 \pm 0,63 \%$, respectivamente .

Foi registrada a redução gradual das alterações morfológicas espermáticas à medida que se ampliava o período de coleta, não havendo diferença

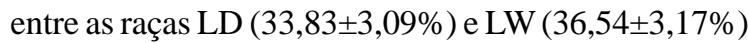
$(\mathrm{P}=0,523)$.

\section{DISCUSSÃO}

O presente trabalho apresenta dados sobre o comportamento e a fisiologia reprodutiva de machos jovens LD e LW empregados como reprodutores em um programa de melhoramento genético nacional. Assim, contribui com informações importantes que servem como indicativo da performance reprodutiva dessas duas raças na fase pós-púbere.

\section{Estudo 1}

O desempenho dos machos frente às fêmeas em estro pode ser considerado ótimo, sendo superior ao observado por NELSSEN et al. (1982), que relata que somente 65\% de machos Yorkshire de 210 dias de idade saltaram sobre fêmeas em estro.

A maioria das informações da literatura fazem referência a diferenças de comportamento sexual existentes entre animais puros e híbridos (NEELY, 1982; IRGANG \& SCHEID, 1992). A ausência de diferenças entre os machos LD e LW no que se refere ao comportamento de monta, pode ser atribuída à semelhança de desenvolvimento corporal e de idade quando testados frente a fêmeas em cio.

Como os padrões de comportamento sexual se desenvolvem durante a fase de crescimento (NEELY, 1982), o contato físico com outros animais nessa fase é fundamental para a expressão da libido em machos jovens (HEMSWORTH et al., 1977; HEMSWORTH \& FINDLAY, 1978; HEMSWORTH et al., 1983, SCHEID \& AFONSO, 2000). Os animais deste experimento foram criados em grupo até o momento de iniciarem o teste. É possível que a oportunidade de adquirir experiência sexual, através do contato físico e das 


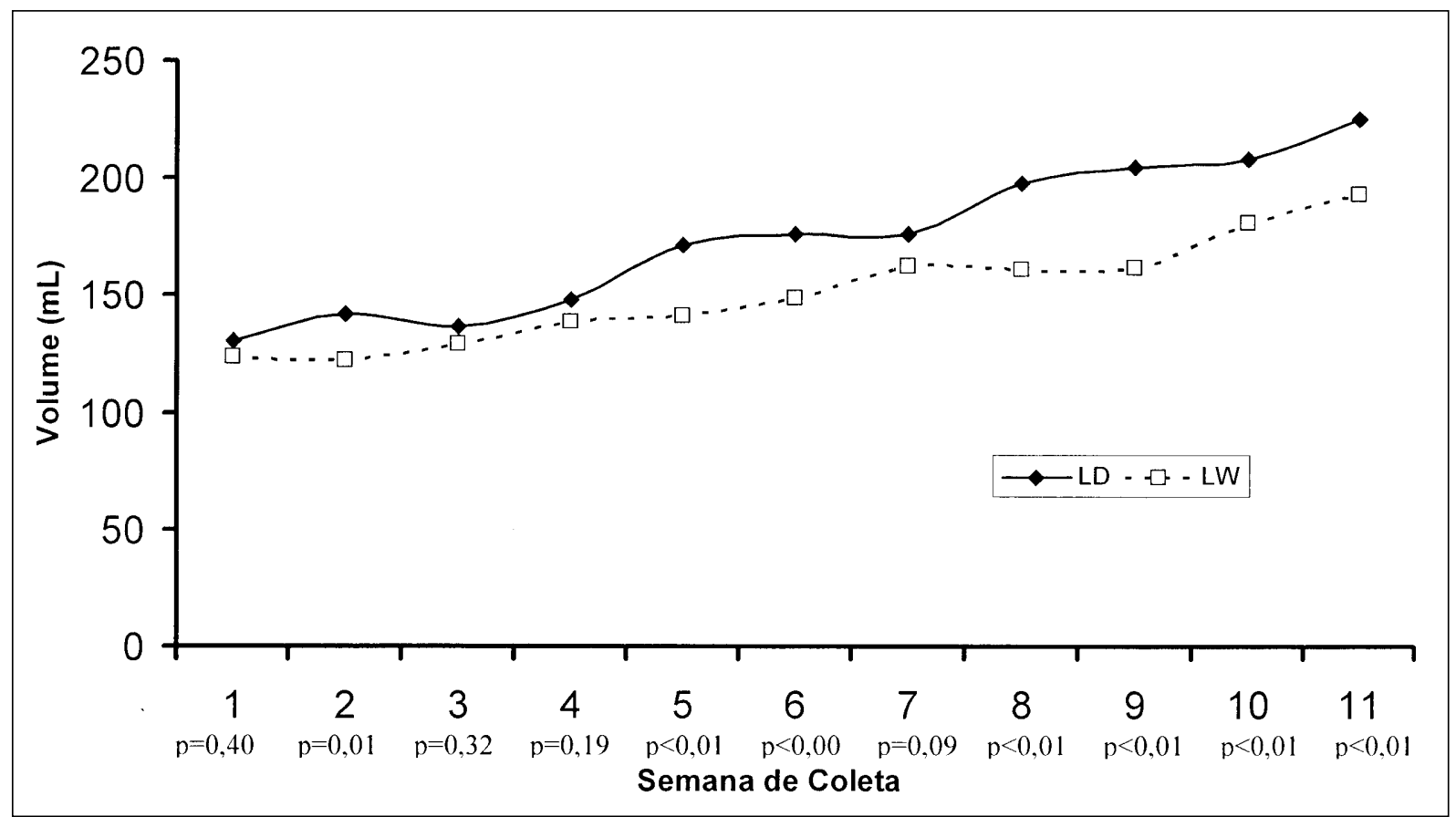

Figura 1- Volume ejaculado pelos machos Landrace (LD) (n=17) e Large White (LW) (n=16), em função da semana de coleta de sêmen ( $\mathrm{P}=$ nível de significância estatística na comparação das médias entre as raças na mesma semana de coleta de sêmen).

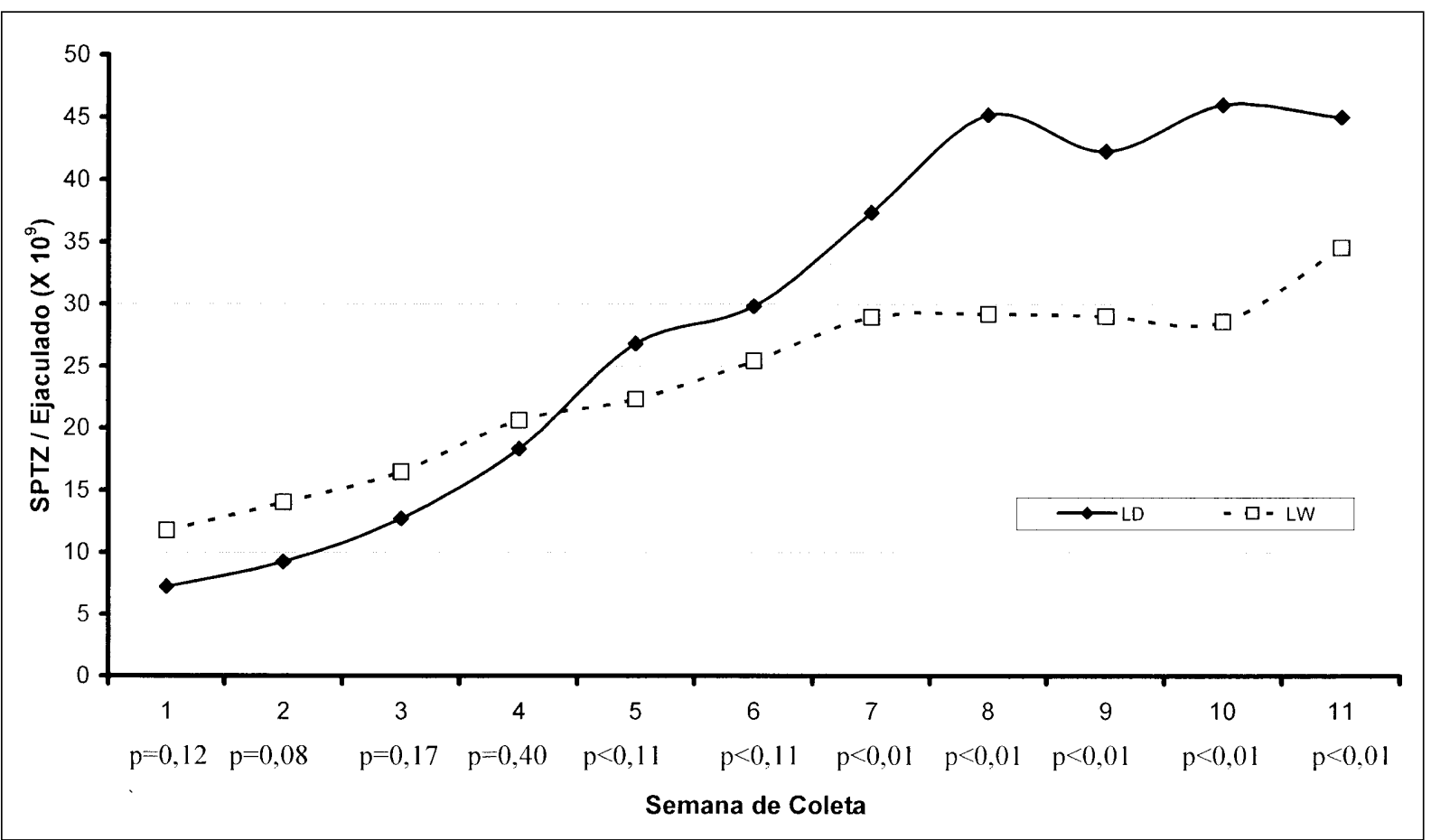

Figura 2 - Médias do número total de espermatozóides no ejaculado dos machos Landrace (LD) (n=17) e Large White (LW) (n=16), em função da semana de coleta de sêmen ( $\mathrm{P}=$ nível de significância estatística na comparação das médias entre as raças na mesma semana de coleta de sêmen).

Ciência Rural, v.35, n.1, jan-fev, 2005. 


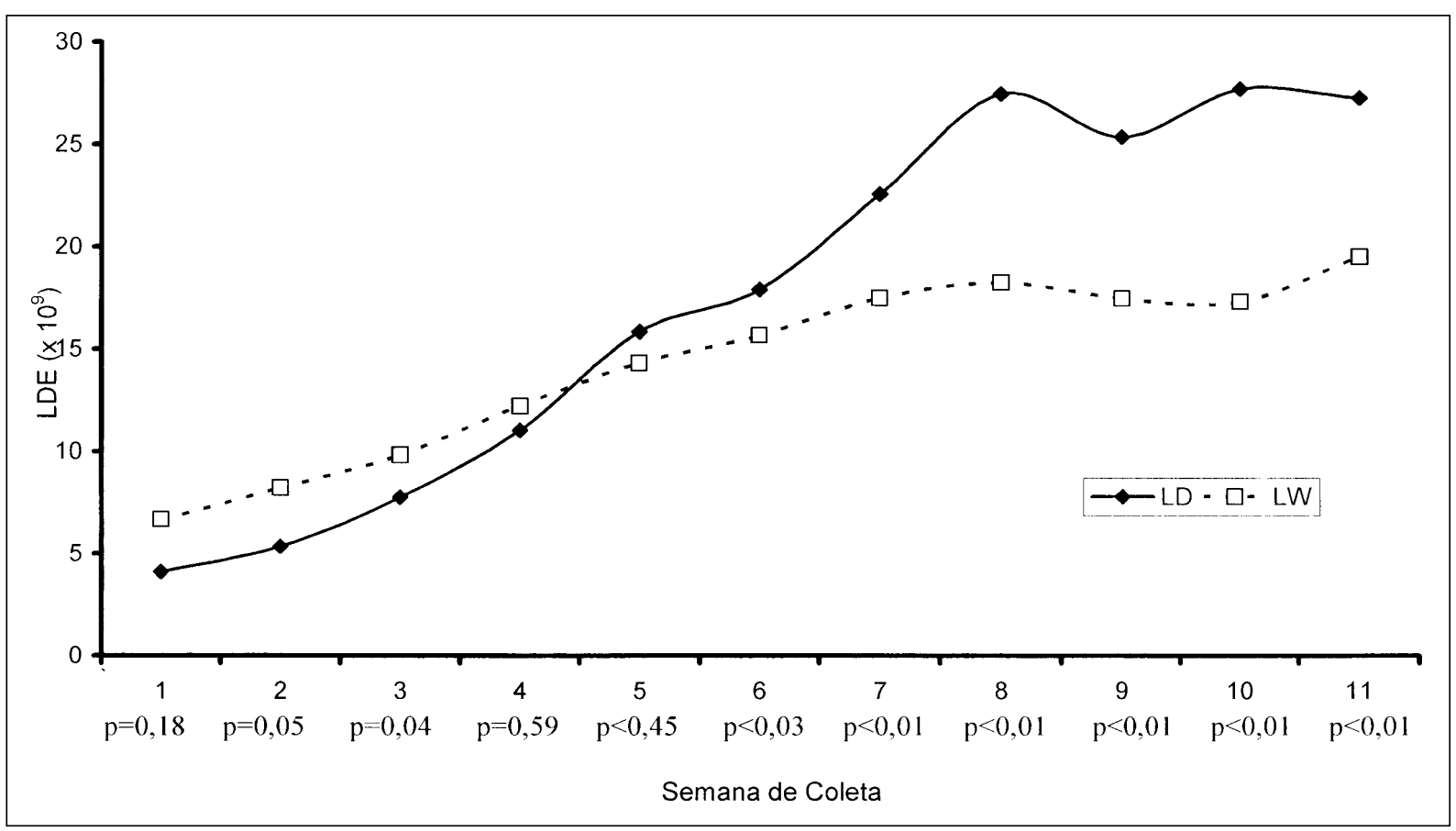

Figura 3 - Liberação diária de espermatozóides (LDE) de machos Landrace (LD) (n=17) e Large White (LW) (n=16), em função da semana de coleta de sêmen ( $\mathrm{P}=$ nível de significância estatística na comparação das médias entre as raças na mesma semana de coleta de sêmen).

interações sociais durante a fase de crescimento, tenha contribuído para a expressão de desempenho favorável durante a avaliação do comportamento de monta.

Os resultados deste estudo sugerem que a libido e a capacidade de monta não são fatores limitantes para o início da vida reprodutiva em machos suínos LD e LW no período estudado.

Estudo 2

Apesar dos animais das duas raças apresentarem pesos e idades similares, foram observadas diferenças em relação às características seminais estudadas. Diferenças raciais foram previamente descritas por outros autores (SWIERSTRA \& RAHNEFELD, 1967; SWIERSTRA, 1973; CONLON \& KENNEDY, 1978; KENNEDY \& WILKINS, 1984, GERFEN et al., 1994).

Os volumes superiores de sêmen ejaculados pelos machos LD estão em concordância com os resultados de SWIERSTRA (1973) e CONLON \& KENNEDY (1978), que relatam que animais LD produzem praticamente o dobro do volume de sêmen, comparativamente a outras raças. No entanto, é interessante ressaltar que a avaliação do desenvolvimento dos órgãos reprodutivos de machos LD e LW com idades entre 150 a 179 dias, não revelou diferenças no comprimento e peso das glândulas bulbouretrais de ambas as raças (ARANTES, 1994). Isso sugere que a diferença na produção de fluídos seminais deva ser atribuída a outros fatores.

Houve um incremento paralelo da concentração espermática e do número de espermatozóides ejaculados, semelhante, nos machos LW, aos valores observados por SWIERSTRA(1966), SWIERSTRA (1973), SWIERSTRA E RAHNEFELD (1967) E SWIERSTRA (1968b) em machos Yorkshire de idades mais avançadas, respectivamente, 270 a 365 dias de idade.

A avaliação da LDE proporciona a estimativa da produção diária de espermatozóides (SWIERSTRA, 1968b; 1971; 1973), representando em tomo de $85 \%$, $88 \%$ ou $83 \%$ desta (SWIERSTRA, 1968a). Os baixos valores do número total de espermatozóides ejaculados e da LDE nas primeiras semanas de coleta são característicos do período póspúbere (FLORCRUZ \& LAPWOOD, 1978). A LDE média do período de coletas, quando os animais das duas raças tinham entre 148 e 232 dias de idade, foi maior que a de machos com idades entre 330 e 540 dias (CAMERON, 1985). Isso confirma que uma maior taxa de crescimento corporal é acompanhada de maior precocidade sexual. Devido à maior capacidade de produção espermática dos machos $\mathrm{LD}$, animais dessa raça podem produzir maior número de doses de sêmen 
quando jovens, o que leva a um aumento na eficiência de uso desses machos em programas de inseminação artificial.

No entanto, nas primeiras coletas foi observado elevado percentual de alterações da morfologia espermática, o que não leva ao descarte, mas inviabiliza o uso dos animais para a reprodução até a normalização desses valores. A alta incidência de patologias espermáticas observada nos animais das duas raças reflete as condições imaturas da espermatogênese (GODINHO E CARDOSO, 1979; SILVA et al., 1999). Sua redução gradativa pode ser atribuída à eficiência do processo espermatogênico e a melhoria das condições de maturação nos epidídimos (MATOS \& THOMAS, 1992).

\section{CONCLUSÕES}

A capacidade de monta parece não ser um fator limitante para o início da vida reprodutiva dos machos Landrace e Large White dentro do período avaliado. Machos Landrace apresentam maior produção espermática e, portanto, potencial para produção de maior número de doses de sêmen quando jovens, comparativamente a machos Large White.

\section{REFERÊNCIAS BIBLIOGRÁFICAS}

ARANTES, V.M. et al. Influência da raça e da idade sobre o peso da carcaça, desenvolvimento sexual e avaliação sensorial da gordura de varrões. In: CONFERÊNCIA INTERNACIONAL SOBRE CIÊNCIA E TECNOLOGIA DE PRODUÇÃO E INDUSTRIALIZAÇÃO DE SUÍNOS, 1., 1995, Campinas, SP. Anais... Campinas : CTC/ITAL, 1995. p. 143.

CAMERON, R.D.A. Measurement of semen production rates of boars. Australian Veterinary Journal, v.62, n.9, p.301304, 1985.

CAMERON, R.D.A. Sexual development and semen production in boars. Pig News and Information, v.8, n.4, p.389-396, 1987.

CONLON, P.D.; KENNEDY, B.W. A comparison of crossbred and purebred boars for semen and reproductive characteristcs. Canadian Journal of Animal Science, v.58, p.63-70, 1978.

FLORCRUZ, S.V.; LAPWOOD, K.R. A longitudinal study of puberal development in boars. International Journal of Andrology, v.1, p.317-330, 1978.

GERFEN, R.W. et al. Comparation of the semen chacacteristics of Fengjing, Meishan and Yorkshire boars. Theriogenology, v.41, p.461-469, 1994.

GODINHO, H.P.; CARDOSO, F.M. Desenvolvimento sexual de porcos Yorkshire. II. Estabelecimento e evolução da espermatogênese. Arquivo da Escola de Veterinária da Universidade Federal de Minas Gerais, v.32, n.3, p.351-361, 1979.
HEMSWORTH, P.H. et al. Influence of social conditions during rearing on the sexual behaviour of the domesticus boar. Animal Production, v.24, p.245-251, 1977.

HEMSWORTH, P.H.; FINDLAY, J.K. The importance of physical contact with other pigs during rearing on the sexual behaviour of the male domestic pig. Animal Production, v.27, p.201-207, 1978.

HEMSWORTH, P.H. et al. The influence of isolation from females and mating frequency on the sexual behaviour and semen quality of young post-pubertal boars. Animal Production, v.37, p.49-52, 1983.

IRGANG, R.; SCHEID, I.R. Association between mating behavior and gonadal traits in purebred and crossbred boars. In CONGRESS INTERNATIONAL PIG VETERINARY SOCIETY, 1992, The Hague, The Netherlands. Proceedings... The Hague, 1992. p.435.

KENNEDY, B.W.; WILKINS, J.N. Boar, breed and enviromental factors influencing semen characteristics of boar used in artificial insemination. Canadian Journal of Animal Science, v.64, p.833-843, 1984.

MATOS, C.A.P.; THOMAS, D.L. Phisiology and genetics of testicular size in sheep: a review. Livestock Production Science, v.32, p.1-30, 1992.

NEELY, J.D. Sexual behaviour and testicular development in purebred and crossbred boars. Dissertation Abstracts International, v.43, n.6, p.1673B, 1982.

NELSSEN, J.L. et al. Reproductive development in young boars exposed to sexually mature, nonpregnant sows and gilts. Theriogenology, v.17, n.5, p.545-550, 1982.

SAS INSTITUTE. SAS user's guide: statistical analysis System, Release 6.12. Cary, NC, 1998.

SCHEID, I.R.; AFONSO, S.B. Treinamento e manejo de machos suínos jovens e adultos. In: SIMPÓSIO INTERNACIONAL DE REPRODUÇÃO E INSEMINAÇÃO ARTIFICIAL EM SUÍNOS, 7., 2000, Foz do Iguaçu. Anais... Piracicaba, 2000. p.29-39.

SILVA, S.M. et al. Estabelecimento da espermatogênese em suínos das raças large white e landrace com diferentes taxas de crescimento dando ênfase à puberdade. In: CONGRESSO BRASILEIRO DE VETERINÁRIOS ESPECIALISTAS EM SUÍNOS, 9., 1999, Belo Horizonte. Anais... Belo Horizonte, 1999. p.301-302.

SWIERSTRA, E.E. Sperm formation and sperm output in Yorkshire and Lacombe boars. Journal of Animal Science, v.25, n.3, p.931, 1966.

SWIERSTRA, E.E. A comparison of spermatozoa production and spermatozoa output of Yorkshire and Lacombe boars. Journal of Reproduction and Fertility, v.17, p.459-469, 1968a.

SWIERSTRA, E.E. Cytology and duration of the cycle of the seminiferous epithelium of the boar; duration of spermatozoan transit through the epididymis. Anatatomical Record, v.161, p.171-186, 1968b. 
SWIERSTRA, E.E. The effect of low ambient temperature on sperm production, epididymal sperm reserves, and semen characteristics of boars. Biology of Reproduction, v.2, p.23-28, 1970 .

SWIERSTRA, E.E. Sperm production of boars as measured rom epididymal sperm reserves and quantitative testicular histology. Journal of Reproduction and Fertility, v.27, p.91-99, 1971.

SWIERSTRA, E.E. Influence of breed, age, and ejaculation frequency on boar semen composition.
Canadian Journal of Animal Science, v.53, p.4353, 1973.

SWIERSTRA, E. E.; DYCK, G.W. Influence of the boar and ejaculation frequency on pregnancy rate and embryonic survival in swine. Journal of Animal Science, v.42, n.2, p.455-460, 1976.

SWIERSTRA, E.E.; RAHNEFELD, G.W. Semen and testis characteristics in young Yorkshire and Lacombe boars. Journal of Animal Science, v.26, p.149-157, 1967. 